\title{
Carnets
}

Revue électronique d'études françaises de l'APEF

Première Série - 2 Numéro Spécial | 2010

Littératures nationales: suite ou fin. Résistances, mutations \& lignes de fuite

\section{Littératures de l'immigration : un pas vers l'interculturalité?}

\section{Anne-Rosine Delbart}

\section{CpenEdition}

\section{Journals}

Édition électronique

URL : http://journals.openedition.org/carnets/5006

DOI : $10.4000 /$ carnets.5006

ISSN : 1646-7698

Éditeur

APEF

Édition imprimée

Date de publication : 1 juin 2010

Pagination : 99-110

\section{Référence électronique}

Anne-Rosine Delbart, «Littératures de l'immigration : un pas vers l'interculturalité? », Carnets [En ligne], Première Série - 2 Numéro Spécial | 2010, mis en ligne le 16 juin 2018, consulté le 10 décembre 2020. URL : http://journals.openedition.org/carnets/5006 ; DOI : https://doi.org/10.4000/carnets.5006

\section{(c) (1) (9)}

Carnets est mis à disposition selon les termes de la licence Creative Commons - Atribution - Pas d'utilisation commerciale 4.0 International. 


\title{
LITTERATURES DE L'IMMIGRATION: \\ un pas vers l'interculturalité?
}

ANNE-ROSINE DELBART

Université Libre de Bruxelles

ardelbar@ulb.ac.be

\begin{abstract}
Résumé
Le choc des langues et des cultures est une si pas la caractéristique première de ce que l'on appelle maintenant de plus en plus souvent au pluriel les littératures francophones. À ce titre, elles constituent un outil précieux pour l'éclairage d'un concept encore trop flou auquel la société, les médias, le monde politique s'intéressent de près: l'interculturalité. Notre contribution voudrait montrer comment les productions littéraires d'immigrés maghrébins de la seconde génération, voire de la troisième génération, tentent un passage de la multiculturalité à l'interculturalité et offrent aussi, par le problème de désignation qu'elles posent (littérature de l'immigration, littérature beure, littérature des quartiers, littérature francophone, littérature française?), une preuve concrète des résistances, des mutations et des lignes de fuite au sein des littératures nationales en ce début de $\mathrm{XXI} \mathrm{l}^{\mathrm{e}}$ siècle.
\end{abstract}

\begin{abstract}
Contact of languages and cultures is the main characteristic of francophone literatures. In that way they are very precious to throw light on the undefined concept of interculturality. Our paper would like to show how the second - even the third - generation of Maghrebian immigrants literature makes concrete the transition of multiculturality to interculturality. The problematic question of its appellation will also give an illustration of resistances, mutations and escapes in national literatures at the beginning of the $\mathrm{XXI}{ }^{\text {st }}$ century.
\end{abstract}

Mots-clés: contact des langues et des cultures, littératures francophones, interculturalité, littérature de l'immigration maghrébine

Keywords: languages and cultures in contact, Francophone literatures, interculturality, Maghrebian immigration literature

Anne-Rosine Delbart, "Litteratures de l'immigration: un pas vers l'interculturalité?", Carnets, Littératures nationales: suite ou fin - résistances, mutations \& lignes de fuite, nº spécial printemps / été, pp. 99-110 http://carnets.web.ua.pt/ 
II n'y a pas d'identité "naturelle" qui s'imposerait à nous par la force des choses. II n'y a que des stratégies identitaires, rationnellement conduites par des acteurs identifiables. Nous ne sommes pas condamnés à demeurer prisonniers de tels sortilèges.

Jean-François Bayart

Réfléchissant au concept d' "exil" dans la préface qu'il a rédigée pour le volume coordonné par Anissa Talahite-Moodley (Problématiques identitaires et discours de l'exil dans les littératures francophones), Charles Bonn constate que l'émigration semble un indicible en littérature (2007: III):

lorsque poussés par une actualité tragique les grands écrivains maghrébins comme Mohammed Dib, Tahar Ben Jelloun ou Rachid Boudjedra parlent enfin de l'émigration/immigration dans la deuxième moitié des années 70 , c'est davantage pour parler de l'exil de l'écriture que de la misère de l'émigré: l'émigration serait-elle un indicible en littérature?

II faudra attendre la seconde moitié des années 80 pour voir se développer une écriture qui se réclame de l'immigration: Azouz Begag (Le gone du Chaâba, 1986), Leïla Houari (Zeida de nulle part, 1985), Ahmed Zitouni (Aimez-vous Brahim?, 1986). Avec Akli Tadjer, on voit apparaître le premier roman drôle sur les immigrés: Les A.N.I. du Tassili en 1984. A.N.I., c'est l'acronyme pour "Arabe Non Identifié", le ton est donné dès le début.

Mais poursuit Bonn, cette littérature de l'immigration maghrébine bouscule "souvent les canons de la littérarité", tout en réussissant, ajouterais-je, à créer, par ce bousculement, un courant nouveau qui a même reçu son étiquetage: Alec Hargreaves a créé, en l'identifiant, le mouvement de la "littérature beure".

En quoi la littérature beure, les littératures de l'immigration, font-elles un pas vers l'interculturalité? Loin de moi l'idée de raviver l'amalgame entre "interculturel" et "immigration" qui a contribué très longtemps au discrédit des études sur l'interculturel. L'interculturalité réussie suppose un cheminement d'entités plurielles l'une vers l'autre. L'art semble être un bon vecteur: voyez la mondialisation par la musique et la chanson anglo-saxonnes. Qu'en est-il pour la littérature? Peut-elle devenir interculturelle et à quel prix? L'interculturalité en littérature nous placerait-elle dans un processus qui conduirait à la fin des littératures nationales pour la grande satisfaction des signataires du Manifeste Pour une littérature- 
monde? Cette question nous place là, me semble-t-il, au coeur des préoccupations des organisateurs du colloque qui ont retenu entre autres, parmi les axes de travail, les questions des "littératures et discours identiaires", des "littératures régionales et marginales", de "la littérature-monde" et des approches inter et multiculturelles qu'elle appelle.

L'interculturalité est un résultat auquel voudraient arriver les sociétés plurielles qui ont affaire aujourd'hui à une population bigarée. L'interculturalité est en tout cas le modèle social privilégié dans le monde français qui diffère du modèle anglais, plaidant, lui, en faveur du multicuturalisme permettant à des communautés culturelles de co-exister sans fusionner.

En Belgique, pour le moment, on réfléchit beaucoup à ces questions. Au mois de septembre 2009, ce sont même ouvertes les assises de l'interculturalité qui encouragent des projets permettant de voir comment gérer la pluralité sociale qui déconcerte.

\section{Construction de l'interculturalité dans les littératures de l'immigration: illustration du processus de "transculturation"}

Il est certain que les ouvrages mettant en scène les conditions de vie des populations émigrées, même de la seconde ou troisième génération, participent d'une démarche qui permet de bâtir l'interculturalité, par un processus que Serge Gruzinski a appelé la "transculturation", le passage (trans-), le cheminement d'une culture à l'autre et retour.

Nous montrerons trois aspects du processus de transculturation qui aboutit à I'interculturalité: (1) les fêtes, (2) l'acceptation de la double appartenance et non plus le rejet de l'une ou de l'autre grâce au retour au pays des ancêtres, et plus largement (3) l'activité d'écriture (la langue et les sujets de l'écriture).

\subsection{Un Noël musulman}

Je puiserai un premier exemple de transculturation dans un livre paru récemment en Belgique sous la plume d'une auteure issue de l'immigration marocaine, Mina Oualdlhadj: Ti t'appelles Aïcha, pas Jouzifine! (Bruxelles, Clepsydre, 2008).

Quelle meilleure mise en mots du processus de transculturation que le chapitre intitulé "Douloureux apprentissages"? Le chapitre illustre les hiatus culturels d'une enfant qui évolue entre deux mondes parallèles qui ne se rencontrent jamais: le monde de la maison et le monde de l'extérieur. L'extrait retenu s'inscrit à la suite d'un développement qui évoque les souvenirs d'écolière de Tamimount, dite Mimi, l'héroïne, confrontée en classe à la fête de la Saint-Nicolas, patron des écoliers en Belgique. Lors de la Saint-Nicolas, qui se fête le 6 
décembre, il est coutume de distribuer des bonbons et des jouets aux enfants, ce que réclame, en vain, la petite Mimi, à ses parents musulmans. Et dans la foulée de la SaintNicolas, Noël se profile:

Je suis rentrée de l'école un premier décembre en chantant: "Saint Nicolas va m'apporter... une poupée, une poupée..." À l'école, on chantait en chœur; à la maison, je chantais toute seule. Six jours durant, j'ai fredonné la même rengaine, ce qui a énervé sérieusement mes parents. Le sixième jour, je suis rentrée avec des mandarines et des spéculoos.

- Regarde, maman, saint Nicolas m'a donné des mandarines!

- C'est bien, ma fille, c'est bon pour la santé, me dit ma mère dans sa langue maternelle.

- Nathalie a reçu une poupée Barbie.

- Et alors?

- Je veux aussi un cadeau!

- Va voir ton père!

Je le trouve allongé devant la télévision.

- Il est où, mon cadeau de Saint-Nicolas?

- Sanicola?

- Oui, il a apporté des cadeaux à tous les enfants de ma classe. Et moi?

Calmement, mon père m'explique dans sa langue que nous sommes des musulmans et que saint Nicolas est chrétien.

- Et alors?

Quand j'insiste, mon père croit que je ne comprends pas sa langue, alors il se met à me parler dans son autre langue, le français marocain.

- SANICOLA IL IXITI PA!

- Mais si, je l'ai vu à l'école! Et moi, j'ai été sage! C'est pas juste!

Dans ces moments-là, mon père ne me parle plus, il agit. II se lève, se penche sur moi en fermant les poings, met son bras de géant autour de ma petite taille, me soulève du sol, se dirige vers la cuisine avec moi qui m'agite sous son bras. II me lâche par terre sans me prévenir et lance à ma mère, en la pointant du doigt:

- Encore un mot...

II ne termine pas sa phrase, ma mère comprend vite. Quant à moi, je capitule.

Quelques semaines plus tard survient l'affaire Père Noël. Je crie à nouveau haut et fort que je veux être comme les autres.

- Je veux un sapin avec des guirlandes! Je veux un sapin avec des guirlandes!

Je fais ma première manif en scandant les mêmes phrases et en chantant: "Petit Papa Noël, quand tu descendras du ciel..."

Mon père reperd patience: 
- PIRSOUN I VA DISCENDRE DI CIEL! ON I DI MOUSLIMAN! TI T'APPILES TAMIMOUNT, TI T'APPILES PAS NATHALIE OU BIRNADITE!

Je comprends une fois de plus ce qu'il dit à ma mère dans leur langue - "Fais-la taire ou je vous étrangle toutes les deux!" - et je prends peur. Mais le lendemain, mon père ramène un tout petit sapin que l'on branche à une prise électrique et qui étincelle de jolies lumières. (Ti t'appelles Aïcha, pas Jouzifine!, 2008: 48-49)

Ce sapin qui clignote dans un foyer musulman est une première manifestation d'un processus de transculturation. Pour l'anecdote, les milieux catholiques regrettent que la fête de Noël, fête de la nativité, ait été pour beaucoup de Chrétiens phagocytées par la célébration païenne anglo-saxonne du Père Noël. Autre manière d'interculturalité...

\subsection{Un "transat" entre deux cultures}

Un autre révélateur de la transculturation exprimée dans les romans de l'immigration, c'est le sentiment de double appartenance plus ou moins tranquille selon les tempéraments, vu comme un enrichissement et non plus un déchirement. Ce sentiment se forge au détour du voyage dans le pays des origines d'où les protagonistes reviennent souvent déçus comme Zeïda dans le roman de Leïla Houari, qui est pour sa famille et ses amis au Maroc, "une étrangère, une petite fille d'Europe":

Étrangère voilà! Elle se sentait tout bonnement étrangère, il n'avait pas suffi de revêtir une blouza, de tirer l'eau du puits pour devenir une autre, tous ils avaient essayé de lui faire plaisir, personne n'a pensé un seul instant qu'elle était sincère, qu'elle voulait effacer, faire une croix sur son passé, non personne n'y a cru et elle avait fini par se convaincre aussi, le choix de s'être retirée totalement de tout ce qui pouvait lui rappeler l'Europe n'avait fait qu'accentuer les contradictions qui l'habitaient. (Zeïda de nulle part, 1985: 74)

Mais comme le dit Habiba Sebkhi (1999), ce voyage "devient la révélation d'une appartenance à l'ici et à l'ailleurs où il ne s'agit pas de choisir une identité, ni "de mettre au diapason deux cultures différentes à force égale, mais plutôt de garder du "sens" pour la culture d'origine dans un contexte d'intégration plus ou moins réussi".

À cet égard, l'image du transat utilisée par Akli Tadjer illustre parfaitement, concrètement le processus de trans-culturation: 
- Culturellement, est-ce que tu as le cul entre deux chaises? Interroge Nelly, qui à n'en point douter commence à glisser doucement mais sûrement vers un profond sommeil. - Tu sais, ma chère, avoir le cul entre la France et l'Algérie, c'est avoir le cul mouillé, et je ne supporte pas d'avoir les fesses mouillées. II y a longtemps que j'ai pigé que pour être bien dans sa peau et à l'aise dans ses babouches, fallait surtout pas choisir entre la France et l'Algérie... D'ailleurs, pourquoi choisir, puisque j'ai les deux... Je ne veux pas être hémiplégique. Mais pour éviter la paralysie d'une partie de mon cerveau il a fallu que j'investisse énormément d'argent... Toutes mes économies... Je me suis acheté un immense transat qui, une fois déplié, s'étale de Tamanrasset à Dunkerque. Mais tu peux me croire, ça n'a pas été une partie de plaisir pour déplier un grand machin comme ça... Maintenant j'ai le privilège de vivre allongé toute l'année la tête face aux soleils... Des vacances à vie si tu préfères. (Les A.N.I. du Tassili, 1984: 174)

\subsection{Un métissage polyfrancophonique}

La littérature beure raconte les itinéraires de vie singuliers de personnes confrontées à la rencontre pas toujours pacifique, pas toujours hostile non plus, d'ailleurs, de langues et de cultures différentes sur un même espace, en adoptant un ton et un langage qui se veut révélateur d'un brassage linguistico-culturel.

Un extrait du roman intitulé Bel-Avenir d'Akli Tadjer explicite ce que certains ont appelé le "taggage linguistique" (Goudaillier, 1997):

C'est vrai que je dois être un des rares, voire le seul, du 75019 à avoir un vocabulaire aussi sophistiqué qu'une tchoutchouka. Dans une même phrase, je peux mélanger du français AOC, de l'argot certifié, de l'arabe cassé, du kabyle dézingué que je saupoudre parfois de verlan périmé. II m'arrive aussi de postillonner quelques mots d'anglais pour faire genre. Genre pas encore has been. (Bel-Avenir, 2006: 57-58)

La récurrence des traits de l'écriture tant thématiques que linguistiques a même permis d'inclure dans ce champ des auteurs qui ne sont pas d'origine maghrébine, qui ne vivent pas dans les cités ou les banlieues françaises, qui ne sont donc pas beurs. C'est ainsi que Daniel Delas note, dans l'article "Particularismes" du Vocabulaire des études francophones, cette réflexion particulièrement stimulante quand on réfléchit au concept d' "interculturalité":

Ce qu'on a appelé le français des cités ou des banlieues est certes caractérisé par le procédé argotique du verlan qu'on retrouve dans la tradition littéraire française depuis 
François Villon mais le lexique et l'accent sont influencés (avec une nette domination de l'influence arabophone) par les langues maternelles des familles des enfants des cités. Là aussi les écrits littéraires, beurs ou de quelque nom qu'on les désigne, jouent un rôle important pour créer une visibilité langagière à ce phénomène. On se trouve là doublement dans les marges de l'espace francophone et il est intéressant que les écrivains qui s'illustrent dans cette écriture soient certes parfois originaires de ces milieux (Azouz Begag par exemple) mais parfois issus d'une mouvance idéologique française (comme Thierry Jonquet ou Paul Smaïl [pseudonyme de Jack-Alain Léger]). Illustration d'un métissage polyfrancophonique en voie de développement.(Delas: 2005: 148)

Voyez comment Paul Smaïl définit son identité de lettré beur à Barbès au début de Vivre me tue:

\section{- Appelez-moi Smaïl.}

J'ai insisté, en détachant bien le i tréma du a. Cela faisait longtemps que je n'avais pas prononcé mon nom en arabe. Ou, pour mieux dire, craché mon nom à l'arabe, raclé au fond de ma gorge le s, le $\mathrm{m}$, le a, le i tréma, le I. D'ordinaire je triche un peu, je prononce à l'anglaise, j'aspire à l'anglaise, avec chic, en souriant: Smile. À quoi cela tient! II me semble alors qu'à l'autre bout du fil, l'employeur sollicité sourit aussi. Et comme mon père eut la bonne idée de me donner un prénom chrétien très banal en déclarant ma naissance à la mairie du $18^{\mathrm{e}}$, le 5 mai 1970, je peux faire illusion en me présentant ainsi. Ensuite, il ne s'agit plus que de débiter sans reprendre haleine mon inutile cursus: bac plus cinq, oui. Oui, Lettres. Littérature comparée... Ah ah! Déjà le sourire se fige à l'autre bout du fil. Ne parlons pas de CV: je ne vais pas énumérer les trois ou quatre petits boulots que j'ai trouvés en attendant... En attendant quoi? Puisque, bien entendu, il n'y aura pas de vrai travail pour moi, soit que mes études ne me qualifient en rien pour le poste proposé, soit que parvenu par la ruse, tréma gommé, photo retouchée, jusqu'à obtenir un entretien, je doive enfin me présenter en chair et en os... En bougnoule, autrement dit. En bicot, en beur, en arbi, en craoui, en rat, en raton, en sidi, en nardène, en melon - au choix. (Vivre me tue, 1997: 11)

Vivre me tue était en fait une fausse autobiographie. C'est en 2003 dans le livre On en est là que Léger révèle le nouveau jeu de cache-cache identitaire auquel il s'est livré en prenant un pseudonyme arabisant et le masque d'un jeune homme issu des banlieues.

Selon Delas, il ne faudrait plus être beur pour parler "beur" ou parler des beurs. Voilà bien une démarche interculturelle si l'on se reporte à l'idée que défend Martine Abdallah- 
Pretceille (dans son "Que sais-je?" sur L'éducation interculturelle): le préfixe "inter" dans "interculturalité" indique, d'une part, une mise en relation entre des groupes, des individus, des identités. Jack-Alain Léger par le choix de son pseudonyme hybride chrétien et musulman, par le sujet de son roman entreprend une mise en relation des identités. Tout le roman renvoie en effet à la manière dont on voit l'Autre et à la manière dont on se voit. Très troublant, très rimbaldien... quand on se dit que l'Autre, c'est moi.

Quoi qu'il en soit, la littérature beure se révèle bien une littérature interculturelle. Le déchainement de la critique quand la révélation de l'identité vraie de l'auteur de Vivre me tue fut connue montre toutefois que la société française n'en est pas encore là dans son cheminement sur l'interculturalité.

II faut qu'un Beur soit un Beur, un Juif un Juif, et eux, bons Français, de bons Français, tolérants, ouverts aux autres. Comme ce professeur de Moustafa, aux Beaux-Arts, qui, bien intentionné, forcément bien intentionné, lui suggère d'illustrer le Coran. Ah oui? Pourquoi? Parce qu'il s'appelle Moustafa? Mais si lui, Moustafa, préfère lire Dostoïevski? (On en est là, 2003: 182-183)

Et inversement quand un beur se pique de sortir d'une thématique beur la critique sort ses griffes. C'est d'une manière plus large un peu le dictat qui pèse sur tous les auteurs en situation de contact des langues et des cultures.

\section{Une désignation problématique}

L'exemple de Smaïl-Léger montre que l'étiquette de littérature beure enferme les auteurs dans des schémas pré-établis qu'ils soient littéraires (la littérature beure raconte les itinéraires de vie singuliers de personnes confrontées à la rencontre de langues et de cultures différentes sur un même espace, en adoptant un ton et un langage qui se veut révélateur d'un brassage linguistico-culturel) ou sociaux (il suffit d'être beur pour faire de la littérature beure) dont ils ne pourraient sortir et situe par là-même l'activité littéraire dans une espèce de communautarisme, une "ghettoisation", disait Régine Robin (Le roman mémoriel, 1989).

Comme le rappelle Anissa Talahite-Moodley dans sa contribution au volume Problématiques identitaires et discours de l'exil dans les littératures francophones consacrée à Azouz Begag et plus particulièrement à son roman intitulé Le marteau pique-cœur, la dénomination littérature beure tombe en désuétude: 
la notion d'une littérature ou d'une culture "beur" (ou "beure" comme on trouve parfois) a servi surtout à souligner l'idée de biculturalisme présent dans cette expression, de synthétisme culturel et de volonté de s'affirmer comme un être hybride, produit de plusieurs cultures. Aujourd'hui, la notion même d'une littérature "beur" semble être tombée en désuétude, laissant ainsi entrevoir un vide qui reflète bien la problématique identitaire dans le contexte de l'immigration maghrébine en France. (2007: 152)

"Inclassable", "innommable" même selon les dires de Charles Bonn ou d'Alec Hargreaves, cette littérature veut se situer hors des étiquetages culturels ou ethniques.

Le problème de désignation que pose cette mouvance littéraire ("littérature de l'immigration", "littérature beure", "littérature des quartiers", "littérature francophone", "littérature française"?) est bien une preuve concrète des résistances, des mutations et des lignes de fuite au sein des littératures nationales en ce début de $\mathrm{XXI}^{\mathrm{e}}$ siècle.

La question est d'autant plus d'actualité que la France s'interroge aujourd'hui sur son identité nationale. Et l'on note dans ce débat que la langue et la littérature participent pour certains Français de leur identité. Fadela Amara, la Secrétaire d'État chargée de la politique de la ville, conçoit toutefois que cette langue et cette littérature ne doivent pas craindre une ouverture sur des pratiques plurielles du langage. Elle rejoint ainsi la conception polyfrancophonique énoncée par Delas que nous avons rappelée plus haut.

Ces productions littéraires beures semblent donc miner de l'intérieur le concept de littérature nationale, entendez ici littérature française, comme le mine de l'extérieur cette fois les auteurs venus d'ailleurs, tous ces auteurs non français qui prennent la plume en français et publient en France, qu'ils soient ou non de langue maternelle française et qu'on rattache au champ de la ou des littératures francophones.

Alors, face à ces phénomènes littéraires relativement nouveaux, quelle dénomination privilégier? La récente proposition d'une littérature-monde participe explicitement de la volonté de délier le pacte langue-nation et s'affiche comme le substitut idéal à la trop contestée étiquette de littérature francophone qui "entérine une ségrégation" (Le Bris, 2007: 45). Pour Nimrod, la notion de "littérature-monde" se substitue d'ailleurs à celle d' "universel" (Pour une littérature-monde, 2007: 233).

Si elle veut se substituer au flou de l'adjectif francophone, l'épithète monde adjointe à littérature, ne permet pourtant pas de gagner en précision. La littérature-monde explicite un souhait d'ouverture, de connexion entre les auteurs, les oeuvres et les langues. Elle plaide en faveur d'un téléscopage des cultures, d'une hybridation d'évidence dans un monde 
polyphonique. Et se fait bien sûr, par là, l'aboutissement littéraire de la théorie du ToutMonde de Glissant.

Mais cette fuite en avant terminologique occulte l'essentiel: la langue française. Nul ne parlait "le francophone" (Tahar Ben Jelloun, Le Monde diplomatique, mai 2007: 21) et personne n'écrit encore "le mondial". Les initiateurs du projet regrettent pour la littérature le diktat de la langue qui a chassé le récit du littéraire ("Pour le sens, voir la forme", Jean Rouaud, 2007: 16), mais il ne faudrait pas pour autant verser dans l'excès inverse et nier la langue qui est le matériau de base de l'artiste écrivain. On ne peut gommer ce critère d'identification premier. Et le rajout d'ailleurs indispensable de "en français" ou "en langue française" des écrivains à "littérature-monde" (Mabanckou, 2007: 65) montre bien les limites de l'efficacité de cette étiquette: elle est lourde et peu économe s'il faut lui rajouter la précision linguistique; elle est trop économe en l'état et désigne alors de fait l'ensemble de la production écrite dans le monde entier, auquel cas, le concept ne signe pas seulement l'acte de décès de la littérature francophone, mais aussi des autres littératures identifiées par la langue dans lesquelles elles s'expriment (la littérature anglaise, la littérature hispanoaméricaine, etc.) et renvoie globalement à l'art littéraire.

La solution que je proposerais dans la foulée des contributeurs de Pour une littérature-monde serait de délier le pacte littérature-nation et lui substituer un pacte unissant une langue et une littérature. II s'agirait de dépasser les littératures nationales et de reconnaître l'étiquette de littérature française pour toutes les littératures qui s'écrive en français, un français bigarré certes par les alluvions diverses venues des quatre coins du monde où la francophonie s'est exportée.

Adopter l'appellation littérature française est un choix théorique, loin d'être partagé par tous, parce qu'il est perçu soit comme un processus de recolonisation mentale, soit comme une servilité masquée sous une hypostasie de la langue française. Les détracteurs de cette étiquette y voient une soumission des écrivains et de leurs productions littéraires à la mère patrie française. Aussi au nom d'une fierté bien légitime, préfèrent-ils renoncer à leur identité linguistique française et accréditer de la sorte eux-mêmes l'idée qu'il ne serait "de bon bec que de Paris", en participant ainsi de première main et bien involontairement, au déclassement des variations régionales du français. Ils hiérarchisent, tout en condamnant les hiérarchies, les variétés de français.

Les productions littéraires intra-hexagonales mais de factures plurielles ou interculturelles cherchent encore leur dénomination si les appellations littératures beures ou littératures de l'immigration deviennent obsolètes, les productions extra-hexagonales ne pourraient recevoir, elles, que l'appellation francophone, à côté de la seule littérature française légitime dans sa désignation, la littérature de France. 
La globalisation inscrite dans le concept de littérature-monde - dont j'ai souligné la trop grande extension plus haut - a le mérite de tenter une unification, de chercher un décloisonnement quand le chemin pris par les spécialistes des littératures en français, loin d'une unité, s'oriente plutôt vers la multiplicité. On comprend bien l'origine de cette démultiplication: au moment où les littératures des pays de langue maternelle française se sont emparées de l'appellation littérature francophone, la nécessité d'une nouvelle étiquette s'est imposée: celle de littératures francophones désormais au pluriel. La francophonie littéraire, terme incluant la pluralité en lui-même, a peu à peu commencé à s'écrire aussi au pluriel.

La question de l'étiquette n'est pas du tout superficielle, contrairement à ce que prétendent dans Pour une littérature-monde Nancy Huston, Maryse Condé ou encore, avec beaucoup d'humour, Fabienne Kanor:

Suis-je un auteur créolofrancophone qui s'ignore? Une écrivaine négropolitanophone? Francopéricophone? Négroparigophone? Francophone? (2007: 241)

II serait illusoire de penser que l'école, la critique, le monde scientifique puissent explorer sans l'identifier l'objet littéraire. Les dénominations de la littérature conditionnent les données universitaires (les champs disciplinaires, les postes d'enseignants-chercheurs, les unités de recherches), les pratiques pédagogiques (l'introduction des auteurs dans les classes sera toute différente selon l'étiquette par laquelle on les désigne) et, de fait, les réalités sociales.

Si les sociétés de culture française veulent travailler à la reconnaissance de l'interculturalité, qu'elles ne se privent pas de la richesse interculturelle de la littérature française d'aujourd'hui en France et hors de France. 


\section{Bibliographie}

Abdallah-Pretceille, Martine (1999). L'éducation interculturelle. Paris, PUF, coll. "Que sais-je?".

BENIAMINO, M., GAUVIN, Lise (2005). Vocabulaire des études francophones. Limoges, PULIM, coll. Francophonies.

DelBART, Anne-Rosine (2005). Les exilés du langage. Limoges, PULIM, coll. Francophonies.

DELBART, Anne-Rosine, (à paraître), "Littérature en français: je dis ton nom. Une littérature-monde face au dilemme irrésolu entre la littérature française et la ou les littérature(s) francophone(s)?', Alger-Paris, co-édition AUF.

GOUDAILLIER, J.-P. (1997). "Pratiques langagières identitaires des cités contemporaines et institution scolaire”. In G. Langouët (dir.), L'état de l'enfance en France, Paris, Hachette, pp. 279-296.

GRUZINSKI, Serge (1999). La pensée métisse. Paris, Fayard.

HOUARI, Leïla (1985). Zeïda de nulle part. Paris, L'Harmattan, coll. Écritures arabes.

LEGER, Jack-Alain (2003). On en est là. Paris, Denoël.

OuAldLHADJ, Mina (2008). Ti t'appelles Aïcha, pas Jouzifine!. Bruxelles, Clepsydre.

SEBKHI, Habiba (1999). "Une "littérature naturelle": le cas de la littérature "beure". In: Itinéraires et contacts de cultures, n27, pp. 16 -27 (consulté sur le site www.limag.refer.org)

RoBIN, Régine (1989). Le roman mémoriel: de l'histoire à l'écriture du hors-lieu. Montréal, Le Préambule, coll. L'univers des discours.

SMAïL, Paul (1997). Vivre me tue. Paris, Balland (rééd. J'ai lu, 1998).

TADJER, Akli (1984). Les A.N.I. du Tassili. Paris, Seuil.

TADJER, Akli (2006). Bel-Avenir. Paris, Flammarion.

TALAHITE-Moodley, Anissa (2007). Problématiques identitaires et discours de l'exil dans les littératures francophones. Ottawa, Presses de l'Université d'Ottawa. 